\title{
A study of pericardial effusion in HIV positive patients and its correlation with the CD4 count
}

\author{
(1) Sharath Madhyastha P. ${ }^{1}$, (1) Charan Thej Reddy ${ }^{1}$, (1) Ganesh V. Shetty ${ }^{1}$, (i) Barkur Ananthakrishna Shastry ${ }^{1}$, \\ (1) Akhila Doddamani²
}

${ }^{1}$ Manipal Academy of Higher Education, Kasturba Medical College, Department of Medicine, Manipal, India

2Manipal Academy of Higher Education, Kasturba Medical College, Department of Community Medicine, Manipal, India

\section{Date submitted:}

15.01.2020

Date accepted:

10.08.2020

Online publication date:

15.03.2021

\section{Corresponding Author:}

Sharath Madhyastha P., Assistant Prof., Manipal Academy of Higher Education, Kasturba Medical College, Department of Medicine, Manipal, India

dr.sharathymc@gmail.com

ORCID:

orcid.org/0000-0003-2821-3473

Keywords: HIV, pericardial effusion, CD4 count, tuberculosis

\begin{abstract}
Aims: Cardiovascular diseases in human immunodeficiency virus (HIV)-positive patients are becoming increasingly detected in developing countries and pericardial effusion is one of the common problems amongst them. The relation of CD4 count is also important, as it correlates with the severity of cardiac involvement. The present study was therefore undertaken to determine the clinical profile of pericardial effusion in HIV patients and its correlation with CD4 count.

Methods: This cross-sectional study was conducted in a tertiary care hospital from 2015 to 2017 and it included 500 HIV patients, 39 of whom had pericardial effusion. All patients were assessed clinically and had undergone electrocardiograph, echocardiography and CD4 count along with the routine investigations. The presence of pericardial effusion was determined by echocardiography. The pericardial fluid was analyzed for cells, biochemistry, Gram staining, AFB staining and cultures. An attempt was made to correlate the findings with CD4 count.
\end{abstract}

Results: Out of 500 HIV-positive patients, 39 (7.8\%) were found to have pericardial effusion. Majority were male (64.1\%) and middle-aged (40-65 y/o) (59\%). Most of the effusions (61.54\%) were quantified as large and the most common etiology was tuberculous (41\%). Twenty-one patients (53.85\%) were in the subgroup of CD4 count $<50$ cells/ $\mu \mathrm{L}$ and 16 of them had severe pericardial effusion. Sixty-four percent of the patients underwent pericardial drainage and the remaining were managed medically.

Conclusions: In the present study, $7.8 \%$ of HIV-infected patients had pericardial effusion. Tuberculosis is the most common cause. The lower the CD4 count is, the larger the pericardial effusion will be.

\section{Introduction}

Pericardial disease was the most frequent clinical manifestation of cardiac disease in patients with human immunodeficiency virus (HIV) infection and, specifically, acquired immunodeficiency syndrome (AIDS). However, in developed countries with widespread access to antiretroviral therapy (ART), the incidence of symptomatic pericardial disease in HIV positive patients has declined significantly. Pericardial effusion was mainly observed in patients with AIDS $(1,2)$. In resource-limited settings, the prevalence of pericardial disease in patients with HIV is similar to that reported from pre-ART studies (3). Nevertheless, cardiac involvement in HIVIAIDS was often underdiagnosed or attributed wrongly to other noncardiac diseases. This is because symptoms like fatigue or reduced exercise tolerance are common in this population (1). Tuberculosis and viral infections are the most common causes of pericardial disease in developing countries (4). The normal CD4 count for most of the laboratories falls in a range of 800 to 1,050 cells $/ \mu \mathrm{L}$. A CD4 count of less than 200 cells $/ \mu \mathrm{L}$ indicates the clinical stage of AIDS. Previous studies have shown that HIV related cardiac abnormalities are more frequently encountered in patients with low CD4 count. Improvement in CD4 count in response to ART has been shown to be the most important predictor of clinical outcome (5). 
The present study was therefore undertaken to determine the clinical profile of pericardial effusion in HIV infection and its correlation with CD4 count.

\section{Methods}

This study was a cross-sectional study conducted in a training and research hospital in Manipal, India, for a period of one year and eight months (September 2015 - May 2017. This study included 500 HIV positive patients, 39 of whom had pericardial effusion. Institutional Ethics Committee, Kasturba Medical College (September 09, 2015, number: IEC 486/2015) approval was sought and granted. All the patients were informed in detail about the study and their written consent was obtained.

Inclusion criteria were HIV-positive patients aged more than 18 years with echocardiographic evidence of pericardial effusion. Development of pericardial effusion following cardiopulmonary surgery or post-myocardial infarction were excluded from the study.

The presenting symptoms of all the patients were recorded. All participants underwent detailed clinical examination with special attention to the cardiovascular system. Complete blood counts, liver \& kidney function tests, CD4 count, chest radiograph, electrocardiograph (ECG) and 2D echocardiogram were performed for all the participants. The pericardial fluid was analyzed for Gram staining, Ziehl-Neelson (Z-N) staining, Gene-Xpert, cytology (cell count, differential counts and malignant cells), biochemistry (protein, albumin, glucose, lactic acid dehydrogenase, adenosine deaminase and $\mathrm{pH}$ ), culture and antibiotic sensitivity. Miscellaneous investigations like thyroid function test (T3, T4 and thyroid stimulating hormone), antinuclear antibody (ANA) and anti-cyclic citrullinated peptide were done in a few selected patients. And finally, the association between the severity of pericardial effusion and CD4 count was assessed.

\section{Statistical Analysis}

All the collected data were entered in a Microsoft Excel sheet and then analyzed using SPSS software (version 15.0, SPSS South Asia Bangalore). Qualitative data were presented as frequency and percentages and analyzed using the chisquare test or Fisher's exact test (in case of $2 \times 2$ contingency tables). Quantitative data were presented as mean and standard deviation. Statistical significance was defined as $p<0.05$.

\section{Results}

Out of 500 HIV patients, 39 patients $(7.8 \%)$ had pericardial effusion. The majority of the study population were males (64.1\%) and belonged to the age group of $41-50$ years (51.28\%). Dyspnea or breathlessness is the most common presenting complaint among the study participants $(92.3 \%)$ followed by palpitations $(64.1 \%)$ and cough $(48.7 \%)$. On cardiovascular examination, the common findings noted were tachycardia (61.5\%) followed by elevated jugular venous pressure (JVP) (41\%) and hypotension $(25.6 \%)$. Chest X-ray showed cardiomegaly in $74.4 \%$. Around one-half of the patients $(51.3 \%)$ had sinus tachycardia followed by low voltage complexes (20.5\%). Out of 39 patients, 24 $(61.54 \%)$ had severe pericardial effusion, $9(23.08 \%)$ had moderate and $6(15.38 \%)$ patients had mild pericardial effusion. More than half $(53.85 \%)$ of the study participants had a CD4 count of less than 50 cells/ $\mu \mathrm{L}, 10$ (25.64\%) patients had CD4 count $50-200$ cells/ $\mu \mathrm{L}$ and $8(20.51 \%)$ patients had CD4 count of more than 200 cells/ $\mu \mathrm{L}$ (Table 1 ).

The most common etiology for pericardial effusion was tuberculosis (41\%) followed by idiopathic/viral (15.4\%), malignant (15.4\%), purulent (10.3), chronic kidney disease (10.3), connective tissue disease (5.1) and hypothyroidism $(2.6 \%)$ (Table 2). Sixty-four percent of the patients underwent pericardial drainage and the remaining were managed medically.

As evident from Table 3, out of 39 participants, 21 patients (53.85\%) were in the subgroup of CD4 count less than 50 cells/ $\mu \mathrm{L}$ and the majority of them presented with severe pericardial effusion $(41.02 \%)$. Whereas only eight patients $(20.51 \%)$ had CD4 count of more than $200 / \mu \mathrm{L}$ and half of them (four patients) had only mild effusion. The difference was statistically significant with a p-value of 0.029 .

\section{Discussion}

Pericardial effusion was described as one of the common cardiac manifestations in HIV infected patients. But the number of HIV patients developing pericardial effusion is decreasing dramatically in developed countries after the introduction of Antiretroviral therapy (1). However, the incidence in resourcelimited settings is similar to that reported in pre-ART studies (6). Sliwa et al. (7) conducted a study in South Africa, which included 518 HIV patients and reported a $25 \%$ prevalence of pericardial disease. However, our study showed that only $7.8 \%$ of patients with HIV had pericardial effusion, as all of them are on ART.

Symptoms range from asymptomatic to severe dyspnea of cardiac tamponade depending on the amount and rate of pericardial effusion. Among symptomatic patients, approximately one-third present with cardiac tamponade $(8,9)$. Only a few cases of acute cardiac tamponade present with classical Beck's triad, which includes low blood pressure (hypotension), elevated JVP and muffled or absent heart sounds on auscultation. One more important finding of cardiac tamponade is pulsus paradoxus. It is defined as a fall in systolic blood pressure of more than 10 $\mathrm{mm}$ of $\mathrm{Hg}$ during inspiration, which is nothing but exaggerated normal physiology (10). In our study, the most common cardiac symptom was breathlessness $(92.3 \%)$, followed by palpitations $(64.1 \%)$ and cough $(48.7 \%)$. On examination, the common cardiac manifestations included tachycardia $(61.5 \%)$ followed by elevated JVP (41\%) and hypotension (25.6\%). 


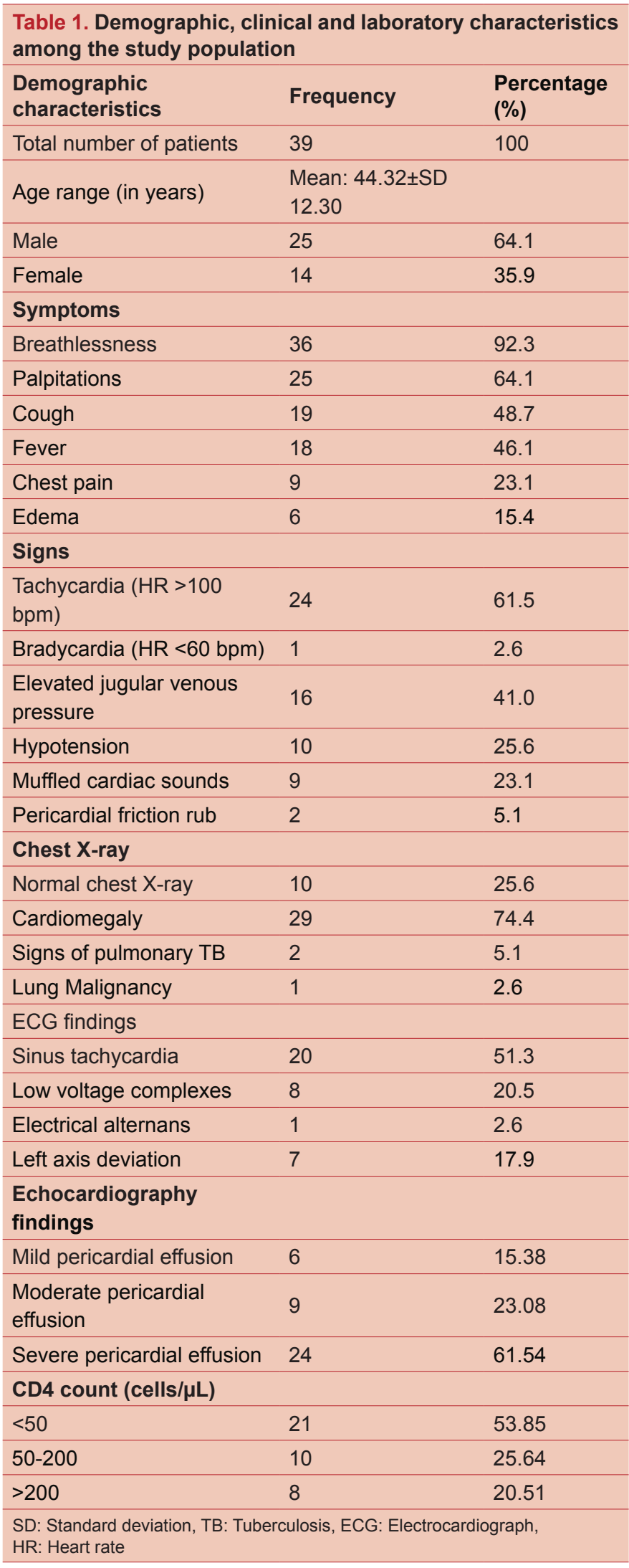

\begin{tabular}{|lcc|}
\hline \multicolumn{3}{l}{ Table 2. Etiological profile of pericardial effusion } \\
\hline Etiologies & Frequency & $\%$ \\
\hline Tuberculosis & 16 & 41.0 \\
\hline Idiopathic/viral & 6 & 15.4 \\
\hline Malignancy & 6 & 15.4 \\
\hline Purulent & 4 & 10.3 \\
\hline CKD & 4 & 10.3 \\
\hline CTD (SLE) & 2 & 5.1 \\
\hline Hypothyroidism & 1 & 2.6 \\
\hline $\begin{array}{l}\text { CKD: Chronic kidney disease, CTD: Connective tissue disease, SLE: Systemic } \\
\text { lupus erythematosus }\end{array}$
\end{tabular}

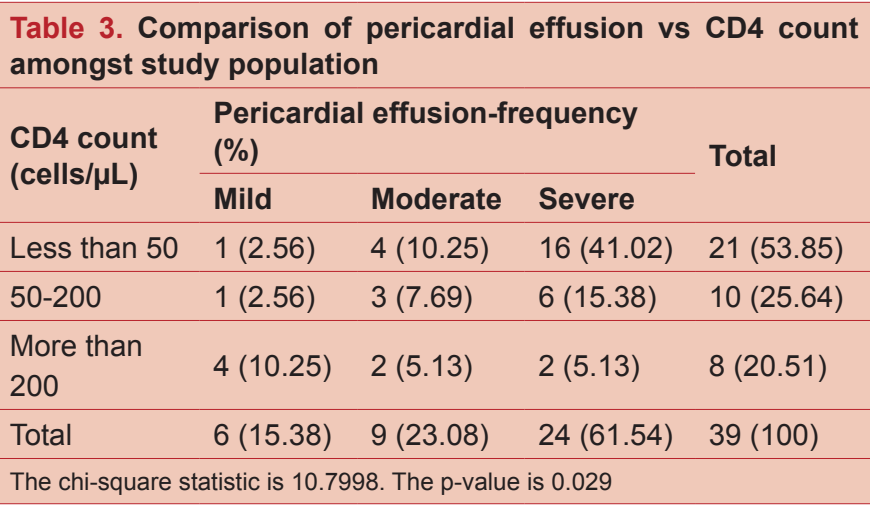

The ECG changes seen in pericardial effusion are low voltage, diffuse ST-segment elevation and total electrical alternans. ST-segment elevation occurs because of the involvement of myocardial inflammation. Total electrical alternans refers to the alternating high and low voltages of all ECG waveforms between cardiac cycles within a given lead due to swinging of the heart in the pericardial fluid $(11,12)$. Low voltage QRS complex is said to be present when the total amplitude of the QRS complex is less than $5 \mathrm{~mm}(0.5 \mathrm{mV})$ in limb leads (lead I, II, III, aVL, aVF, aVR) and QRS amplitude less than $10 \mathrm{~mm}(1$ $\mathrm{mV}$ ) in chest leads (V1 to V6). Pericardial effusion with cardiac tamponade should be suspected when there is a combination of low voltage complexes and sinus tachycardia in the ECG (13). In the present study, the most common abnormal ECG finding was sinus tachycardia $(51.3 \%)$ followed by low voltage complexes $(20.5 \%)$. One patient with massive pericardial effusion had total electrical alternans.

The findings on chest X-ray are inconsistent, based on the etiology and size of the effusion and underlying cardiac illness. Effusion volume less than 200 to $300 \mathrm{~mL}$ (small to moderate effusion) may not be visible in chest X-ray at all, while larger pericardial effusion is classically present with an enlarged cardiac silhouette. Lung fields are usually clear unless there is an associated heart failure. However, these findings on chest X-ray are neither sensitive nor specific for the diagnosis of pericardial effusion (14). In the present study, two-third of the study population was found to have cardiomegaly in chest $\mathrm{X}$-ray. 
The pericardial effusion is usually graded into mild, moderate and severe using echocardiography and it is measured in diastole. When the effusion is seen only posteriorly with a thickness of less than $10 \mathrm{~mm}$, it is classified as mild effusion (50 to $100 \mathrm{~mL}$ ). Moderate effusion (100 to $500 \mathrm{~mL}$ ) is seen along the length of the posterior wall but not anteriorly and the thickness is 10 to $20 \mathrm{~mm}$. If the effusion is seen circumferentially and the thickness greater than $20 \mathrm{~mm}$, it is said to be large (>500 mL) (14). In the present study, 24 patients $(61.54 \%)$ presented with large pericardial effusion.

Among asymptomatic patients, most of the time, the exact etiology of pericardial effusion is not known or not identified as they rarely require pericardiocentesis. Infection and neoplasm constitute approximately two-thirds of the causes among symptomatic patients (2). Infectious causes vary with geography. In developing countries, tuberculosis is the most common cause of pericardial effusion. By contrast, in resourcerich settings, less than 5 percent of pericarditis in HIV is due to tuberculosis $(15,16)$. Other causes for pericardial effusion in HIV patients include Staphylococcus aureus, Streptococcus pneumoniae, Listeria, Chlamydia species, Cryptococcus, Nocardia, Aspergillus and neoplasms such as lymphoma and Kaposi's sarcoma (KS) $(17,18)$. Even in our study, the most common etiology for pericardial effusion was tuberculosis (41\%) followed by idiopathic/viral (15.4\%), malignant (15.4\%) and purulent $(10.3 \%)$ effusion. Other rare causes we encountered were chronic kidney disease $(10.3 \%)$, connective tissue disease $(5.1 \%)$ and hypothyroidism (2.6\%). Two patients with connective tissue disease had systemic lupus erythematosus and both of them had high titers of ANA and anti-ds DNA.

The most common cause of malignant pericardial effusion in HIV positive patients is AIDS-related KS. However, with the advent of potent ART, the incidence of $K S$ has reduced significantly. Other common primary tumours involving the pericardium are lung, breast and esophageal malignancies $(8,19)$. In the present study, out of 6 malignant pericardial effusions, three patients had lung cancer, two patients had breast cancer and the remaining one patient had AIDS-related KS.

The treatment of pericardial effusion in patients with HIV varies depending on the severity and etiology of the disease. Asymptomatic patients with small pericardial effusion without tamponade require no intervention, but they should be followed-up to look for any progression of the disease. By contrast, symptomatic patients with a large effusion require pericardiocentesis and further treatment. Cardiac tamponade is a medical emergency and requires immediate drainage $(20,21)$. Further treatment is warranted based on the identified or suspected etiology. For example, anti-tubercular therapy is indicated in tubercular pericardial effusion $(22,23)$. Purulent pericardial effusion is treated with intravenous antimicrobial therapy. The empirical antibiotic therapy should cover both Gram- positive and Gram-negative bacterial pathogens. The most commonly used antibiotics are Vancomycin plus $3^{\text {rd }}$ generation cephalosporins like Ceftriaxone or a carbapenem such as imipenem. Intravenous antibiotic therapy should be continued for approximately two to four weeks until the resolution of all clinical signs of infection. Cardiac tamponade can be drained either by pericardiocentesis (i.e. echocardiography guided percutaneous drainage) or by surgical drainage. Both are highly effective in the removal of fluid and relief of symptoms related to hemodynamic compromise. According to the 2015 European Society of Cardiology guidelines on pericardial effusion, catheter pericardiocentesis is the preferred treatment in most patients. An indwelling catheter is usually placed in the pericardial space until the daily pericardial fluid drain is less than $25 \mathrm{~mL}$. Whereas, neoplastic effusion requires an extended period of drainage (14). In the present study, $64 \%$ of the patients underwent pericardial drainage and the remaining cases were managed medically.

The occurrence of pericardial effusion in an HIV positive patient is generally considered as a poor prognostic sign. Previous studies have shown that HIV related cardiac abnormalities are more frequently encountered in patients with low CD4 count. There are several potential explanations existing for this observation. Low CD4 count suggests the state of severe immunosuppression and development of pericardial effusion may be a marker for undiagnosed opportunistic infections (5). Previous studies have reported that in patients with AIDS with pericardial effusion, CD4 counts were significantly lower than in those without effusion. The pericardial effusion with tamponade in HIV patients is associated with low CD4 count and may be a marker of end-stage infection $(18,19,21)$. Even in our study, 21 patients had a CD4 count of less than 50 cells/ $\mu \mathrm{L}$ and the majority of them (16 patients) presented with severe pericardial effusion.

The sample size of the present study is relatively small. The exact etiology of mild and few moderate pericardial effusions were uncertain as they were managed conservatively without pericardiocentesis. Larger follow-up studies are required for a better understanding of pericardial effusion complications and survival amongst HIV positive patients.

\section{Conclusion}

In the present study, $7.8 \%$ of HIV-infected patients had pericardial effusion. Tuberculosis is the most common cause. The management of pericardial effusion was directed at the etiology and one-third of them were managed conservatively. The lower the CD4 count is, the larger the pericardial effusion will be.

\section{Acknowledgments}

We acknowledge the department of cardiology for providing echocardiography reports. 


\section{Ethics}

Ethics Committee Approval: Approval from the Institutional Ethics Committee, Kasturba Medical College (September 09, 2015, number: IEC 486/2015) was sought and granted.

Informed Consent: Written informed consent was obtained from all the participants.

Peer-review: Externally and internally peer-reviewed.

\section{Authorship Contributions}

Concept: B.A.S., S.M.P., Design: G.V.S., Data Collection or Processing: C.T.R., G.V.S., Analysis or Interpretation: S.M.P., A.D., Literature Search: C.T.R., S.M.P., Writing: S.M.P., C.T.R.

Conflict of interest: No conflict of interest was declared by the authors.

Financial Disclosure: The authors declared that this study received no financial support.

\section{References}

1. Lind A, Reinsch N, Neuhaus K, et al. Pericardial effusion of HIV-infected patients ? Results of a prospective multicenter cohort study in the era of antiretroviral therapy. Eur $\mathrm{J}$ Med Res. 2011;16:480-483.

2. Estok L, Wallach F. Cardiac tamponade in a patient with AIDS: a review of pericardial disease in patients with HIV infection. Mt Sinai J Med. 1998;65:33-39.

3. Thienemann F, Sliwa K, Rockstroh JK. HIV and the heart: the impact of antiretroviral therapy: a global perspective. Eur Heart J. 2013;34:3538-3546.

4. LeWinter M, Kabbani S. Pericardial diseases. In: Zipes DP, Libby P, Bonow RO BE, edd. Braunwald's Heart Disease. 7th ed. Philadelphia, Pa: Elsevier Saunders; 2005:17571780.

5. Mishra TK, Mishra SK, Singh S. Cardiovascular manifestations of people living with HIVIAIDS: Report from a hot spot in eastern India. Indian Heart J. 2019;71:338-343.

6. Ntsekhe M, Mayosi BM. Cardiac manifestations of HIV infection: an African perspective. Nat Clin Pract Cardiovasc Med. 2009;6:120-127.

7. Sliwa K, Carrington MJ, Becker A, Thienemann F, Ntsekhe $\mathrm{M}$, Stewart S. Contribution of the human immunodeficiency virus/acquired immunodeficiency syndrome epidemic to de novo presentations of heart disease in the Heart of Soweto Study cohort. Eur Heart J. 2012;33:866-874.

8. Chen Y, Brennessel D, Walters J, Johnson M, Rosner F, Raza M. Human immunodeficiency virus-associated pericardial effusion: report of 40 cases and review of the literature. Am Heart J. 1999;137:516-521.

9. Eisenberg MJ, Gordon AS, Schiller NB. HIV-associated pericardial effusions. Chest. 1992;102:956-958.
10. Beck CS. Two cardiac compression triads. J Am Med Assoc. 1935;104:714-716.

11. Wagner G, Strauss D. Marriott's Practical Electrocardiography. 12th ed. Lippincott Williams \& Wilkins; 2014:263-267.

12. Nizet PM, Marriott HJ. The electrocardiogram and pericardial effusion. JAMA. 1966;198:169-170.

13. Bruch C, Schmermund A, Dagres $N$, et al. Changes in QRS voltage in cardiac tamponade and pericardial effusion: reversibility after pericardiocentesis and after anti-inflammatory drug treatment. J Am Coll Cardiol. 2001;38:219-226.

14. Adler $Y$, Charron P, Imazio M, et al. 2015 ESC Guidelines for the diagnosis and management of pericardial diseases: The Task Force for the Diagnosis and Management of Pericardial Diseases of the European Society of Cardiology (ESC) Endorsed by: The European Association for CardioThoracic Surgery (EACTS). Eur Heart J. 2015;36:29212964.

15. Ntsekhe M, Mayosi BM. Tuberculous pericarditis with and without HIV. Heart Fail Rev. 2013;18:367-373.

16. Reuter $\mathrm{H}$, Burgess LJ, Doubell AF. Epidemiology of pericardial effusions at a large academic hospital in South Africa. Epidemiol Infect. 2005;133:393-399.

17. Gowda RM, Khan IA, Mehta NJ, Gowda MR, Sacchi TJ, Vasavada BC. Cardiac tamponade in patients with human immunodeficiency virus disease. Angiology. 2003;54:469474.

18. Boulanger E, Gérard L, Gabarre J, et al. Prognostic factors and outcome of human herpesvirus 8-associated primary effusion lymphoma in patients with AIDS. J Clin Oncol. 2005;23:4372-4380.

19. Wilkes JD, Fidias P, Vaickus L, Perez RP. Malignancyrelated pericardial effusion. 127 cases from the Roswell Park Cancer Institute. Cancer. 1995;76:1377-1387.

20. Hsia J, Ross AM. Pericardial effusion and pericardiocentesis in human immunodeficiency virus infection. Am J Cardiol. 1994;74:94-96.

21. Blanchard DG, Hagenhoff C, Chow LC, McCann HA, Dittrich HC. Reversibility of cardiac abnormalities in human immunodeficiency virus (HIV)-infected individuals: a serial echocardiographic study. J Am Coll Cardiol. 1991;17:12701276.

22. Syed FF, Mayosi BM. A modern approach to tuberculous pericarditis. Prog Cardiovasc Dis. 2007;50:218-236.

23. Maisch B, Seferović PM, Ristić AD, et al. Guidelines on the diagnosis and management of pericardial diseases executive summary; The Task force on the diagnosis and management of pericardial diseases of the European society of cardiology. Eur Heart J. 2004;25:587-610. 